\title{
El aumento asimétrico del diámetro de la arteria pulmonar no siempre es secundario a hipertensión arterial pulmonar
}

\section{Pulmonary artery asymmetric enlargement is not always secondary to pulmonary hypertension}

\author{
Carlos García, MD. ${ }^{(1)}$; LUISA Martínez, MD. ${ }^{(2)}$; CAMILA Ospina, MD. ${ }^{(3)}$
}

\begin{abstract}
Resumen
La hipertensión pulmonar hace referencia a un grupo de desórdenes con etiología variable, en los cuales hay aumento de la presión en la arteria pulmonar, hecho que incrementa el diámetro de este vaso. Se presenta el caso de un hombre de 27 años, con diagnóstico de virus de inmunodeficiencia humana, a quien se le detectó, mediante escanografía, aumento del calibre de la arteria pulmonar y su rama izquierda. Inicialmente se consideró hipertensión arterial pulmonar asociada a virus de inmunodeficiencia humana, pero en estudios ecocardiográficos posteriores no hubo evidencia de alteraciones. Se decidió, entonces, realizar una resonancia magnética cardiaca funcional en la que se confirmó hipertensión pulmonar secundaria a estenosis supravalvular, la cual condiciona la asimetría en las ramas de la arteria pulmonar. Este hallazgo justifica la importancia de considerar aspectos clínicos en concordancia con los estudios radiológicos y ecocardiográficos para argumentar la realización de métodos imagenológicos alternativos.
\end{abstract}

Palabras clave: hipertensión arterial pulmonar, hipertensión arterial pulmonar asociada a virus de inmunodeficiencia humana, resonancia magnética cardiaca funcional.

\begin{abstract}
Pulmonary hypertension refers to a group of disorders of variable etiology in which there is elevation of pressure in the pulmonary artery, a fact that increases the diameter of this vessel. We present the case of a 27 -year-old man, diagnosed with HIV infection, in whom increased caliber of the pulmonary artery and its left branch was documented by CT-scan. HIV-associated pulmonary arterial hypertension was first considered, but echocardiograms did not give evidence of any alteration. It was then decided to perform a functional cardiac magnetic resonance, which showed pulmonary hypertension secondary to supravalvular stenosis, thus explaining the asymmetry of the branches of the pulmonary artery. This finding justifies the importance of considering clinical aspects together with radiologic and echocardiographic studies in order to justify alternative image studies.
\end{abstract}

Keywords: pulmonary arterial hypertension, HIV-associated pulmonary arterial hypertension, functional cardiac magnetic resonance.

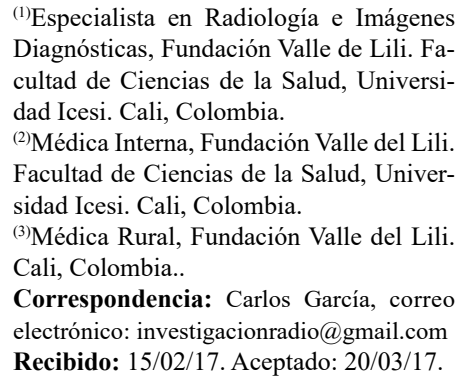

${ }^{(1)}$ Especialista en Radiología e Imágenes Diagnósticas, Fundación Valle de Lili. Facultad de Ciencias de la Salud, Universidad Icesi. Cali, Colombia.

${ }^{(2)}$ Médica Interna, Fundación Valle del Lili. Facultad de Ciencias de la Salud, Universidad Icesi. Cali, Colombia.

${ }^{(3)}$ Médica Rural, Fundación Valle del Lili. Cali, Colombia.

Correspondencia: Carlos García, correo electrónico: investigacionradio@gmail.com Recibido: 15/02/17. Aceptado: 20/03/17. 


\section{Introducción}

La hipertensión pulmonar es una enfermedad en la que se eleva la presión de la arteria pulmonar, y por tanto la resistencia vascular y el diámetro de los vasos pulmonares, generándose hipertrofia ventricular derecha (1). La enfermedad se clasifica en cinco tipos según su etiología; el primero de ellos se denomina hipertensión arterial pulmonar e incluye causas como enfermedades del tejido conectivo, idiopática y virus de inmunodeficiencia humana, entre otras (2). En el caso que se expone, inicialmente se consideró que los hallazgos radiológicos eran secundarios a hipertensión arterial pulmonar asociada a virus de inmunodeficiencia humana.

En los últimos años, gracias al uso de la terapia antirretroviral, la causa de muerte por virus de inmunodeficiencia humana pasó de las infecciones oportunistas a condiciones cardiovasculares como el infarto agudo de miocardio, tumores pericárdicos, miocardiopatías, vasculitis e hipertensión pulmonar (1). La hipertensión arterial pulmonar asociada a virus de inmunodeficiencia humana (HPT-VIH) es una complicación poco común, se presenta en el $0,5 \%$ de la población infectada con VIH, si bien es más común que en la población general (3-5). Respecto a la fisiopatología que genera la HTP-VIH, se han sugerido múltiples teorías; por ejemplo: las proteínas del virus generan daño endotelial y estrés oxidativo, la expresión de endotelina 1 se asocia a hipertensión pulmonar y a una presentación más severa de la enfermedad (5-8). También, se han descrito factores proinflamatorios como citoquinas y factores de crecimiento, como la IL-6 que produce proliferación de células de músculo liso (9).

Se presenta el caso de un paciente masculino de 27 años, con diagnóstico de VIH, a quien, mediante una escanografía de tórax, se revela aumento del diámetro del tronco de la arteria pulmonar y su rama izquierda, por lo que se sospecha HTP-VIH. Sin embargo, en estudios posteriores de ecocardiografía se descarta esta posibilidad. Debido a esta inconsistencia se decide ordenar una resonancia magnética en la que se confirma hipertensión pulmonar causada por estenosis supravalvular, que explica la asimetría en las ramas de la arteria pulmonar y la hipertensión arterial pulmonar.

\section{Caso}

Paciente masculino de 27 años de edad, con antecedentes de carcinoma escamocelular anal, condilomatosis anal y VIH, remitido a nuestra institución para ser valorado por Oncología y Radioterapia. Al ingreso se encontraba en tratamiento antirretroviral desde el 2011. En ese momento refería dolor anal perilesional ocasional y sangrado con la deposición, sin síntomas adicionales, como disnea, tos o edema.

Por antecedente de enfermedad granulomatosa pulmonar previa se tomó una escanografía de tórax donde se evidenció aumento del calibre del tronco de la arteria pulmonar principal y su rama izquierda, que sugería hipertensión arterial pulmonar (figura 1). Se indicó, por consiguiente, toma de ecocardiograma en el cual no se observó aumento de la presión en la arteria pulmonar o signos indirectos de hipertensión pulmonar. Debido a esta

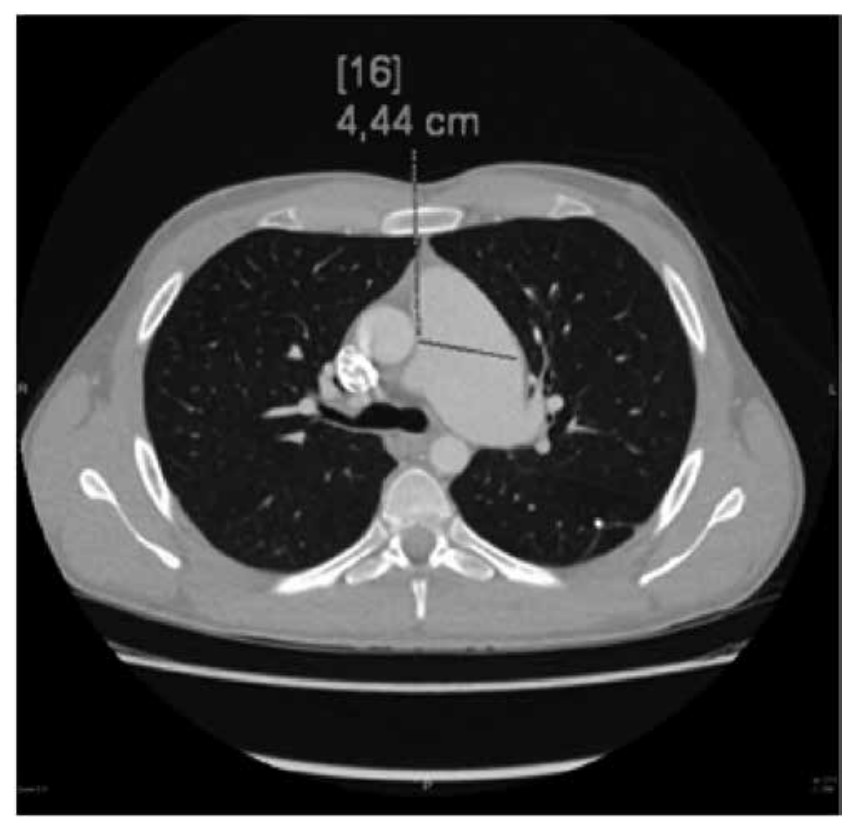

Figura 1. Escanografía de tórax con medio de contraste en corte axial, en la que se evidencia aumento del calibre del tronco de la arteria pulmonar principal. 
incongruencia se efectuó una resonancia magnética funcional de corazón, con el fin de hacer una mejor caracterización; se halló asimetría en el calibre de las arterias pulmonares derecha e izquierda (figura 2) y engrosamiento de la pared del ventrículo derecho, con aumento en la aceleración en la arteria pulmonar principal (figura 3) durante la sístole con gradiente alto y velocidad mayor de $11 \mathrm{~cm} / \mathrm{s}$ en la arteria pulmonar principal (figura 4) (10-13) congruente con hipertensión arterial pulmonar secundaria a estenosis supravalvular, como origen de la asimetría de los vasos pulmonares.

\section{Discusión}

Las enfermedades cardiovasculares se han convertido en la principal causa de muerte en VIH, gracias al uso de la terapia antirretroviral, dejando a un lado las infecciones oportunistas. Por esta razón, los investigadores han enfocado sus estudios en describir la fisiopatología y manejo ideal de la HTP-VIH. Sin embargo, se deben tener en cuenta los diagnósticos diferenciales, ya que no todos los hallazgos de aumento del diámetro de los vasos pulmonares por imagen, son secundarios a esta patología, como se refleja en este caso, donde el aumento del diámetro

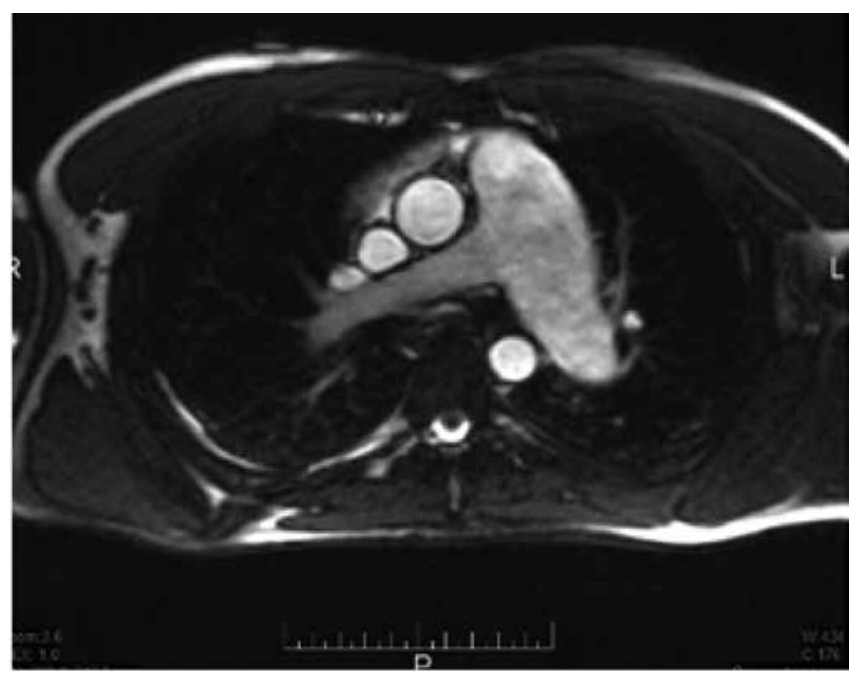

Figura 2. Resonancia magnética de corazón con reconstrucción axial sobre las arterias pulmonares, que muestra asimetría en el calibre de las arterias pulmonares derecha e izquierda. de la arteria y ramas pulmonares es secundario a estenosis supravalvular.

En cuanto a los síntomas con los que se presentan los pacientes de HTP-VIH, son inespecíficos y pueden ser confundidos con otras patologías. Cursan con disnea, edema periférico, tos, fatiga y dolor torácico $(2,14)$. En el caso, el paciente no refería ningún tipo de sintomatología similar que pudiera sugerir un problema cardiovascular; sin embargo, el hallazgo escanográfico del aumento en el calibre de la arteria pulmonar principal mayor de $29 \mathrm{~mm}$, sugirió hipertensión arterial pulmonar como causa (12).

En el diagnóstico el ecocardiograma se usa como herramienta inicial para evaluar las condiciones cardiovasculares asociadas a VIH, debido a su seguridad, disponibilidad y costo-efectividad (15). No obstante, la angiografía sigue siendo el estándar de oro para el diagnóstico de hipertensión pulmonar (16), ya que sólo el 28\% de los casos de HTP-VIH detectados por ecocardiografía son confirmados a través de angiografía (5). Pese a ello, este método es invasivo y supone mayor riesgo para el paciente

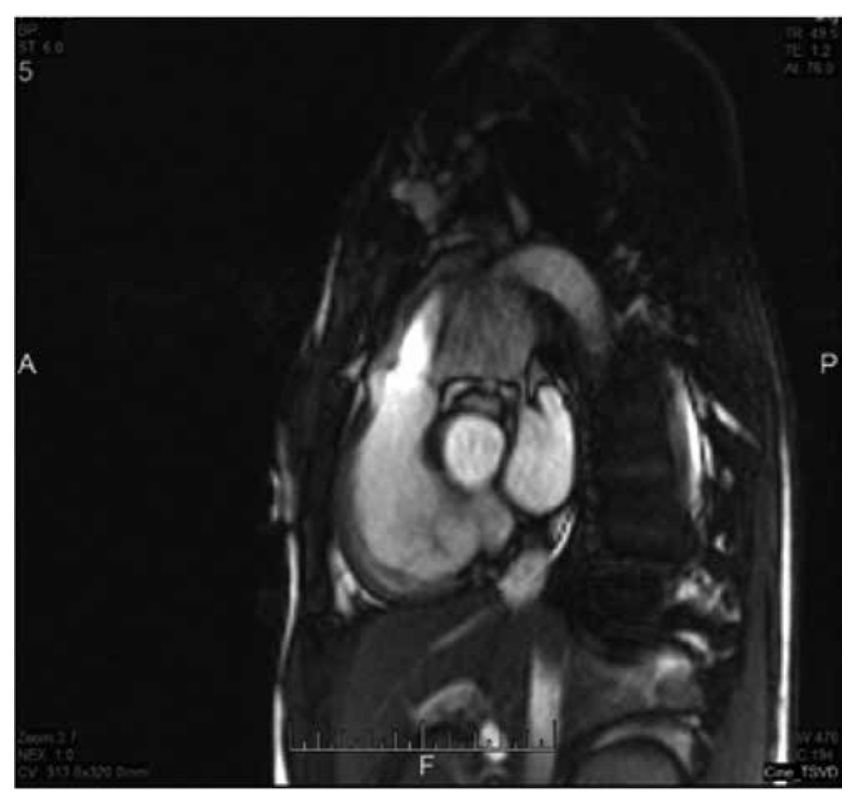

Figura 3. Resonancia magnética de corazón con reconstrucción sagital del tracto de salida del ventrículo derecho, que muestra engrosamiento de la pared del ventrículo derecho, con aumento en la aceleración en la arteria pulmonar principal durante la sístole. 


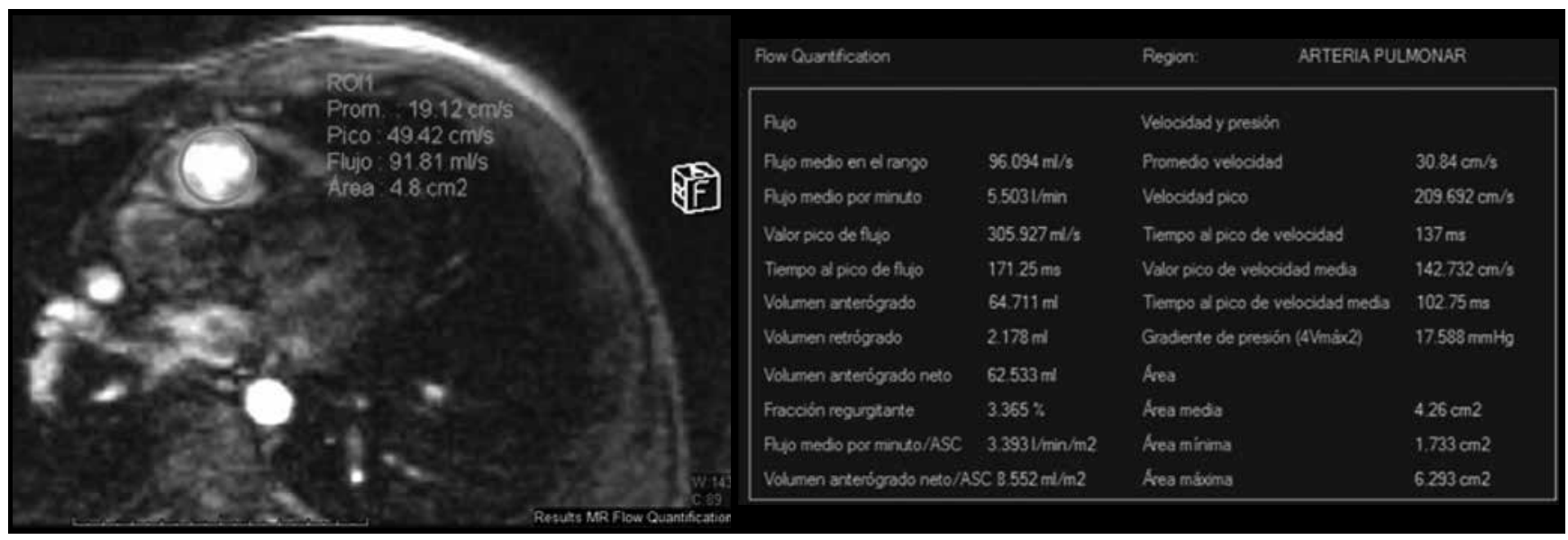

Figura 4. Medida y resultados de la cuantificación del flujo de la arteria pulmonar a través del uso de resonancia magnética cardiaca funcional, que reporta una velocidad promedio aumentada de $19 \mathrm{~cm} / \mathrm{s}$.

(17), de ahí que en los últimos años los estudios se han enfocado hacia la evaluación de otras modalidades, como la tomografía axial computarizada y la resonancia magnética.

Esta última ha adquirido gran importancia por su alta precisión y reproducibilidad. Además, permite visualizar los defectos cardíacos, el parénquima pulmonar y la vasculatura. También se realizan cálculos de volúmenes, como fracción de eyección, y volúmenes de fin de sístole y diástole (18). Adicionalmente, se ha descrito que al conjugar medidas computacionales con los hallazgos en resonancia magnética se pueden reflejar cambios hemodinámicos en la vasculatura pulmonar, proporcionando un método no invasivo que aporta más datos y diagnósticos diferenciales para la evaluación de la hipertensión pulmonar (17).

En la actualidad las guías para el manejo de VIH, no contemplan el tamizaje de rutina de las complicaciones cardiovasculares y pulmonares asociadas a esta(14). Así, cuando se presentan los síntomas mencionados con anterioridad, se debe tener un alto índice de sospecha y evaluar la presencia de alteraciones cardiacas y pulmonares.

Diversas patologías pueden ocasionar aumento del calibre del tronco de la arteria pulmonar en la radiografía de tórax, simulando hipertensión pulmonar
(19) y de ahí la importancia de descartar otros diagnósticos diferenciales, pues el manejo varía en las distintas patologías. Por ejemplo, la embolia pulmonar puede ocasionar aumento del diámetro de la arteria pulmonar, en tanto que la estenosis de la válvula pulmonar y la coartación de la arteria pulmonar, son patologías que aumentan el flujo sanguíneo pulmonar. Adicionalmente, los defectos septales que generan cortocircuito de izquierda a derecha, la falla cardiaca de alto gasto, el síndrome de Marfan y el síndrome de retorno venoso pulmonar anómalo, también pueden ocasionar este hallazgo radiográfico (20). El conjunto de imágenes y la clínica guiarán el diagnostico final. Sin embargo, el aumento asimétrico del diámetro de las arterias pulmonares sugiere otras etiologías como los aneurismas o las estenosis valvulares, que en el caso expuesto requirieron imágenes complementarias de resonancia magnética para aclarar la causa y determinar de manera más exacta la dinámica del flujo pulmonar.

Adicionalmente se debe tener en cuenta que usualmente esta patología se detecta en estadios tardíos de la enfermedad, cuando su pronóstico es pobre y los tratamientos no son efectivos (5), hecho que sugiere que la implementación de tamizaje de individuos con HTP-VIH es necesaria, ya que se podría detectar la enfermedad de manera oportuna y así obtener un mejor pronóstico. 
Finalmente, en este caso la causa del aumento asimétrico del calibre de las arterias pulmonares era secundario a estenosis supravalvular, patología que requiere un tratamiento diferente al de la HTP-VIH.

\section{Conclusiones}

El aumento en el calibre de las arterias pulmonares usualmente es causado por hipertensión arterial pulmonar; sin embargo, cuando es asimétrico sugiere otra etiología como las estenosis valvulares o supravalvulares.

Es importante reconocer la importancia de la concordancia entre la evaluación clínica, radiológica y ecocardiográfica en hipertensión arterial pulmonar, para decidir la realización de otros métodos con mayor desempeño diagnóstico, como la resonancia magnética cardiaca funcional, en especial cuando hay incongruencia entre los métodos diagnósticos anteriores.

\section{Conflictos de interés}

Los autores declaran no tener conflictos de interés.

\section{Bibliografía}

1. Correale M, Palmiotti GA, Lo Storto MM, Montrone D, Foschino Barbaro MP, Di Biase M, et al. HIV-associated pulmonary arterial hypertension: from bedside to the future. Eur J Clin Invest. 2015;45(5):515-28.

2. Mirrakhimov AE, Ali AM, Barbaryan A, Prueksaritanond S. Human immunodeficiency virus and pulmonary arterial hypertension. ISRN Cardiol. 2013;2013:903454.

3. Dai HL, Zhang M, Xiao ZC, Guang XF, Yin XL. Pulmonary arterial hypertension in HIV infection: a concise review. Heart Lung Circ. 2014;23(4):299-302.

4. Triplette M, Crothers K, Attia EF. Non-infectious pulmonary diseases and HIV. Curr HIV/AIDS Rep. 2016;13(3):140-8.

5. Henriques-Forsythe M, Annangi S, Farber HW. Prevalence and hospital discharge status of human immunodeficiency virus-associated pulmonary arterial hypertension in the United States. Pulm Circ. 2015;5(3):506-12.

6. Tcherakian C, Couderc LJ, Humbert M, Godot V, Sitbon O, Devillier P. Inflammatory mechanisms in HIV-associated pul- monary arterial hypertension. Semin Respir Crit Care Med. 2013;34(5):645-53.

7. Bigna JJ, Sime PS, Koulla-Shiro S. HIV related pulmonary arterial hypertension: epidemiology in Africa, physiopathology, and role of antiretroviral treatment. AIDS Res Ther. 2015;12:36.

8. Feijoo MQ, Toro R, López Vazquez de la Torre M, Lennie V, Arce C, Moreno V, et al. Relationship between endothelin-1 levels and pulmonary arterial hypertension in HIV-infected patients. AIDS. 2014;28(18):2693-9.

9. Thao C, Shorr AF, Woods C. Non-infectious pulmonary disorders in HIV. Expert Rev Respir Med. 2017:1-12.

10. Saremi F, Gera A, Ho SY, Hijazi ZM, Sánchez-Quintana D. $\mathrm{CT}$ and MR imaging of the pulmonary valve. Radiographics. 2014;34(1):51-71.

11. Varaprasathan GA, Araoz PA, Higgins CB, Reddy GP. Quantification of flow dynamics in congenital heart disease: applications of velocity-encoded cine MR imaging. Radiographics. 2002;22(4):895-905; discussion -6.

12. Junqueira FP, Lima CM, Coutinho AC, Parente DB, Bittencourt LK, Bessa LG, et al. Pulmonary arterial hypertension: an imaging review comparing MR pulmonary angiography and perfusion with multidetector CT angiography. Br J Radiol. 2012;85(1019):1446-56.

13. Bradlow WM, Gibbs JS, Mohiaddin RH. Cardiovascular magnetic resonance in pulmonary hypertension. J Cardiovasc Magn Reson. 2012;14:6.

14. Schwarze-Zander C, Pabst S, Hammerstingl C, Ohlig J, Wasmuth JC, Boesecke C, et al. Pulmonary hypertension in HIV infection: a prospective echocardiographic study. HIV Med. 2015;16(9):578-82.

15. Pristera N, Musarra R, Schilz R, Hoit BD. The Role of Echocardiography in the Evaluation of Pulmonary Arterial Hypertension. Echocardiography. 2016;33(1):105-16.

16. François CJ, Schiebler ML. Imaging of Pulmonary Hypertension. Radiol Clin North Am. 2016;54(6):1133-49.

17. Lungu A, Swift AJ, Capener D, Kiely D, Hose R, Wild JM. Diagnosis of pulmonary hypertension from magnetic resonance imaging-based computational models and decision tree analysis. Pulm Circ. 2016;6(2):181-90.

18. Pektas A, Olgunturk R, Cevik A, Terlemez S, Kacar E, Oner YA. Magnetic resonance imaging in pediatric pulmonary hypertension. Tex Heart Inst J. 2015;42(3):209-15.

19. FA B. Differential diagnosis in conventional Radiology. Publication NyTm, editor 1991.

20. Reed JC. Chest radiology, patterns and differential diagnoses. Year Book Medical Publishers; 1981. 